\title{
RADIOCARBON, LTD NATURAL RADIOCARBON MEASUREMENTS II
}

\author{
CHARLES S TUCEK
}

\section{Radiocarbon, Ltd, Lampasas, Texas 76550}

Radiocarbon, Ltd moved its laboratory facilities from Spring Valley, New York to Lampasas, Texas in the summer of 1974. This date list represents all results obtained at the Spring Valley location for which sample data have been received, and which have not been reported previously.

Sample preparation techniques and $\mathrm{CO}_{2}$ counting procedures remain as previously reported $(\mathrm{R}, 1971, \mathrm{v} 13$, p 74). In July 1972 we began benzene counting as well as $\mathrm{CO}_{2}$ counting. $\mathrm{CO}_{2}$ is converted to benzene following the procedure of Noakes et al (1966) and scintillation counting is done with a Picker Nuclear Liquimat, optimized for low-level counting. Many samples were counted both as $\mathrm{CO}_{2}$ and as benzene, while the benzene synthesis technique was being perfected. The data obtained (Table 1) indicate good agreement between the 2 methods.

Samples are listed in chronologic order of preparation; relatively poor data for the 1 st 10 samples are a reflection of the learning process in synthesizing benzene. It is interesting that good concordance in the 2nd group of samples is achieved even though \% benzene yield is quite variable, and relatively low at times. Following these cross-check samples, 57 samples were run strictly as benzene, the average yield being $76 \pm 4 \%$.

All samples described below report dates by $\mathrm{CO}_{2}$ counting, except for RL-221, -222, $-223,-298,-300,-302,-310,-318$, and -322 , which were obtained by benzene counting.

Age calculations are based on ${ }^{14} \mathrm{C}$ half-life of 5570 years, using 0.95 NBS oxalic acid as the modern value. Errors are based on counting statistics, and for finite ages are quoted to $\pm 1 \sigma$; for samples approaching the modern or background value, $2 \sigma$ limits are used.

\section{ACKNOWLEDGMENTS}

We are grateful to John Noakes, University of Georgia, and to Don Kearns, Applied Science Technology, Inc, for their help in setting up the benzene operation. We also acknowledge cooperation of our clients who have supplied the sample descriptions and authorized their use, together with the results.

\section{SAMPLE DESCRIPTIONS}

\section{ARCHAEOLOGIC SAMPLES}

\section{A. Alaska}

\section{RL-402. Ester Creek, Alaska}

Ivory from mammoth tusk from Ester Creek; 16km W of Fairbanks, Alaska (ca $65^{\circ} \mathrm{N}, 145^{\circ} \mathrm{W}$ ), from base of ca $32 \mathrm{~m}$ frozen, wind-deposited loess, atop $10 \mathrm{~m}$ alluvial gravels, assoc with many Pleistocene remains. 
TABLE 1

$\mathrm{CO}_{2}-$ Benzene Cross-Check Samples

\begin{tabular}{|c|c|c|c|c|}
\hline Sample no. & $\mathrm{CO}_{2}$ date & Benzene date & $\begin{array}{l}\text { Results } \\
\text { overlap } \\
\text { within }\end{array}$ & $\begin{array}{c}\text { Benzene } \\
\text { yield }(\%)\end{array}$ \\
\hline RL-174 & $4540 \pm 110$ & $4700 \pm 250$ & $1 \sigma$ & - \\
\hline RL-168 & $10,140 \pm 170$ & $9720 \pm 720$ & $1 \sigma$ & - \\
\hline RL-161 & $480 \pm 100$ & $990 \pm 290$ & $2 \sigma$ & - \\
\hline RL-173 & $200 \pm 90$ & $740 \pm 260$ & $2 \sigma$ & - \\
\hline RL-175 & $1490 \pm 100$ & $1860 \pm 180$ & $2 \sigma$ & - \\
\hline RL-176 & $<310$ & $220 \pm 110$ & $1 \sigma$ & 62 \\
\hline RL-172 & $<230$ & $\sim 200$ & $1 \sigma$ & 52 \\
\hline *RL-164 & $1310 \pm 110$ & $1790 \pm 120$ & $3 \sigma$ & 54 \\
\hline * RL-171 & $11,400 \pm 850$ & $8600 \pm 2250$ & $1 \sigma$ & 25 \\
\hline RL-154 & $9700 \pm 620$ & $6340 \pm 1600$ & $2 \sigma$ & 32 \\
\hline *RL-177 & $6150 \pm 120$ & $6150 \pm 150$ & $1 \sigma$ & 62 \\
\hline *RL-178 & $400 \pm 100$ & $490 \pm 100$ & $1 \sigma$ & 62 \\
\hline * RL-179 & $550 \pm 105$ & $450 \pm 120$ & $1 \sigma$ & 51 \\
\hline *RL-180 & $1330 \pm 105$ & $1330 \pm 115$ & $1 \sigma$ & 75 \\
\hline *RL-181 & $1410 \pm 105$ & $1410 \pm 115$ & $1 \sigma$ & 58 \\
\hline RL-185 & $6470 \pm 140$ & $6200 \pm 150$ & $1 \sigma$ & 50 \\
\hline RL-142 & $>31,400$ & $>32,300$ & $1 \sigma$ & 55 \\
\hline RL-182 & $6490 \pm 130$ & $6840 \pm 150$ & $2 \sigma$ & - \\
\hline RL-183 & $11,950 \pm 280$ & $11,700 \pm 300$ & $1 \sigma$ & 68 \\
\hline RL-187 & $330 \pm 90$ & $290 \pm 90$ & $1 \sigma$ & 72 \\
\hline RL-152 & $8320 \pm 220$ & $7620 \pm 520$ & $1 \sigma$ & 29 \\
\hline RL-196 & $340 \pm 90$ & $320 \pm 130$ & $1 \sigma$ & 71 \\
\hline RL-185a & $6470 \pm 140$ & $6450 \pm 130$ & $1 \sigma$ & 84 \\
\hline RL-192 & $380 \pm 80$ & $440 \pm 100$ & $1 \sigma$ & - \\
\hline *RL-224 & $560 \pm 120$ & $580 \pm 100$ & $1 \sigma$ & 78 \\
\hline RL-255 & $1710 \pm 110$ & $1910 \pm 100$ & $1 \sigma$ & 71 \\
\hline RL-217 & $1120 \pm 100$ & $1220 \pm 90$ & $1 \sigma$ & 76 \\
\hline
\end{tabular}

* Descriptions for these samples appear below, results based on $\mathrm{CO}_{2}$ counting.

Coll 1955 by Nick Glumac; subm by Edward Felien, Morgan Park High School, Duluth, Minnesota. Comment: sample was check on another portion of tusk, by high school students Cheryl Doherty and Mary
Richardson.

\section{B. Western United States}

\section{RL-149. Birch Bay, Washington}

$1580 \pm 120$ AD 370

Charcoal from Site $45-\mathrm{WH}-24$, on $10 \mathrm{~m}$ terrace at W margin of Birch Bay $\left(48^{\circ} 56^{\prime} \mathrm{N}, 122^{\circ} 47^{\prime} 25^{\prime \prime} \mathrm{W}\right)$. Sample came from hearth feature at depth 21 to $40 \mathrm{~cm}$, containing finely broken shell and bits of mammal bone. Coll 1969 by C E Larsen and James Edris; subm by G F Grabert, 
Western Washington State Coll, Bellingham, Washington. Comment (GFG): many cobble tools found, typologically different from and assumed to antedate artifacts from shell midden site, $45-\mathrm{WH}-11,400 \mathrm{~m} \mathrm{E}$ on presently forming sand spit beach. Cobble and flake tools are rare in latter site, which yields bone, antler, and ground stone artifacts of typically Marpole through Whalen and more recent aspect. Age of this hearth is probably similar to present and largest midden, but not assoc with cobble implement component.

\section{RL-272. Strait of Georgia, Washington}

$2630 \pm 240$

Charcoal from Site $45-\mathrm{WH}-1$, on wave-cut terrace, at ca $3 \mathrm{~m}$ level, approx N limit of San Juan Is., Washington $\left(48^{\circ} 05^{\prime} 45^{\prime \prime} \mathrm{N}, 122^{\circ} \mathrm{W}\right)$. Sample from base of short trench at depth 160 to $175 \mathrm{~cm}$, from shallow firepit. Coll 1971 by John Prager; subm by G F Grabert. Comment (GFG): sample should date early use of Cherry Point site for human habitation (Grabert \& Larsen, 1973).

\section{Ferndale series, Washington}

Samples from Site $45-\mathrm{WH}-34$, at NE city limit of Ferndale, Washington, right bank of Nooksack R (48 $\left.51^{\prime} 30^{\prime \prime} \mathrm{N}, 122^{\circ} 35^{\prime} \mathrm{W}\right)$. Subm by G F Grabert (Grabert \& Larsen, 1973).

\section{RL-273. No. 249}

$4180 \pm 120$ 2230 BC

Charcoal from intrusive firepit $>1 \mathrm{~m}$ deep, at depth $117 \mathrm{~cm}$ of pit containing abundant shell remains. Coll 1972 by Jean Bosch. Comment (GFG): although $11 \mathrm{~km}$ from salt water, molluscan remains were marine species. Preliminary stratigraphic analysis places intrusive firepit in chronologic relationship with Fraser Delta Marpole phase. Artifacts are similar; many chipped stone projectile points of lanceolate and stemmed forms occur in shell zones and some in non-shell horizons. RL-273 and -274, below, possibly bracket early soil formation and mid-occupation period.

\section{RL-274. No. 511}

$$
1030 \pm 100
$$

Charcoal (bark and wood) from charred log lying on and in paleosol formed on alluvial sand of Nooksack R. Coll 1972 by L K Strickland. Comment (GFG): $\log$ was with a few scattered basalt flake tools and 1 well-formed basalt triangular scraper. Several thin strata of charcoal and dark-stained earth contained a few other artifacts indicating series of brief occupations. Should date early occupation of small remnant levee ridge along small former channel of Nooksack $R$.

\section{RL-275. No. 586}

$$
1210 \pm 100
$$

\section{AD 740}

Charcoal from area of RL-274, from sloping edge of intrusive depression, vertically and horizontally distinct from firepit with shell that yielded RL-273. Coll 1972 by L K Strickland. Comment (GFG): should 

date what may be a large semisubterranean dwelling, relatively rare in
region.

General Comment (GFG): all 3 samples in series came from trench $20 \mathrm{~m}$ $1 \times 2 \mathrm{~m}$ w. Its $\mathrm{E}$ end yielded RL-273 in deep and presumably late prehistoric pit; RL-275 came from 2nd depression extending from ca 8 to $18.5 \mathrm{~m} \mathrm{~W}$ of ref. RL-274 lay outside depression, and seems stratigraphically earlier. Samples should determine construction chronology, occupation date of depression, and relationship to cultural phases of Fraser Delta, and Gulf Is. Assoc antler sedges, projectile points, and many small bone implements in fill suggest woodworking and fishing activities.

\section{Clover Valley Wash series, Nevada}

Clover Valley Wash is ca $26 \mathrm{~km}$ ESE of Caliente $\left(37^{\circ} 31^{\prime} \mathrm{N}, 114^{\circ}\right.$ $16^{\prime} \mathrm{W}$ ), at alt ca $1630 \mathrm{~m}$. Samples coll 1970 by D B Madsen, Univ Missouri, Columbia and P J Mehringer, Jr; subm by D D Fowler, Desert Research Inst, Univ Nevada, Reno.

\section{RL-104. Fright Midden No. 2}

$250 \pm 90$

Juniper twigs (Juniperus osteoperma) from consolidated fossil woodrat midden from small cliff alcove overlooking Clover Valley Wash.

\section{RL-105. Fright Midden No. 1} cliff alcove overlooking Clover Valley Wash.

\section{Meadow Valley Wash series, Nevada}

Samples from various sites $S$ of Caliente, Nevada. 1st group of 8 coll 1971 by D B Madsen and P J Mehringer, Jr; subm by D D Fowler.

RL-126. No. 1

Charcoal from Stein Canyon Rock $24 \mathrm{~km} \mathrm{~S}$ of Caliente $37^{\circ}$ Can lowest $15 \mathrm{~cm}$ of lowest $15 \mathrm{~cm}$ of basal cultural unit at rock shelter; 4 to $4.15 \mathrm{~m}$ below surface.

\section{RL-127. No. 2}

$890 \pm 150$

Charcoal, $100 \mathrm{~m} \mathrm{~S}$ of ranch pump pond, ca $11 \mathrm{~km} \mathrm{~S}$ of Caliente $\left(37^{\circ}\right.$ $\left.34^{\prime} \mathrm{N}, 114^{\circ} 34^{\prime} \mathrm{W}\right)$. Scattered charcoal from base of fill of channel cut in Meadow Valley Wash alluvium. Comment: $\mathrm{NaOH}$ pretreatment to
remove humic acids.

RL-128. No. 3

Charcoal, $100 \mathrm{~m}$ N of mouth of Picnic Canyon, ca $13 \mathrm{~km} \mathrm{~S}$ of Caliente $\left(37^{\circ} 33^{\prime} \mathrm{N}, 114^{\circ} 36^{\prime} \mathrm{W}\right)$. Scattered charcoal from base of fill of higher of the 2 channel cuts in Meadow Valley Wash alluvium. Comment: $\mathrm{NaOH}$ pretreatment to remove humic acids. 
RL-129. No. 4

$400 \pm 150$

Charcoal, ca $100 \mathrm{~m} \mathrm{~N}$ of Etna, Nevada $\left(37^{\circ} 35^{\prime} \mathrm{N}, 114^{\circ} 35^{\prime} \mathrm{W}\right)$. Scattered charcoal from lowest peat band in series of pond deposits in Meadow Valley Wash alluvium. Comment: $\mathrm{NaOH}$ pretreatment to remove humic acids.

RL-130. No. 5

$$
\begin{array}{r}
1670 \pm 130 \\
\text { AD } 280
\end{array}
$$

Seeds (Cyperaceae sp) 200m N of S boundary of Conway Ranch; ca $.8 \mathrm{~km} \mathrm{NE}$ of Etna, Nevada $\left(37^{\circ} 35^{\prime} \mathrm{N}, 114^{\circ} 34^{\prime} \mathrm{W}\right)$. Scattered sedge seeds from lowest peat band in pond sediments in Meadow Valley Wash alluvium. Comment: $\mathrm{NaOH}$ pretreatment to remove humic acids.

\section{RL-165. No. 6}

$$
1240 \pm 150
$$$$
\text { AD } 710
$$

Charcoal from same site as RL-130. Scattered charcoal from lower $25 \mathrm{~cm}$ of pinkish-gray sand stratum overlying peat bands at Loc I, Meadow Valley Wash.

\section{RL-166. No. 7}

$420 \pm 190$

Charcoal from same site as RL-128. Scattered charcoal from base of fill of lower of the 2 channel cuts in Meadow Valley Wash alluvium.

\section{RL-167. No. 8}

AD 1440

$510 \pm 220$

Charcoal, $.8 \mathrm{~km} \mathrm{~N}$ of trestle; ca $20.6 \mathrm{~km} \mathrm{~S}$ of Caliente $\left(37^{\circ} 27^{\prime} \mathrm{N}\right.$, $\left.114^{\circ} 34^{\prime} \mathrm{W}\right)$. Scattered charcoal from lowest $(10 \mathrm{~cm})$ peat bands in Meadow Valley Wash alluvium at Loc IV. Second group of 5 samples coll 1972 and subm by $\mathrm{D}$ B Madsen.

\section{RL-219. No. 9}

$3190 \pm 140$

Charcoal from E arroyo wall; ca $8.2 \mathrm{~km} \mathrm{~S}$ of Caliente $\left(37^{\circ} 33^{\prime} 20^{\prime \prime} \mathrm{N}\right.$, $\left.114^{\circ} 33^{\prime} 50^{\prime \prime} \mathrm{W}\right)$. Scattered charcoal throughout Unit B at Loc VII in Meadow Valley Wash. Unit B is stratigraphically 2nd depositional unit in Meadow Valley Wash alluvium.

\section{RL-220. No. 10}

$2430 \pm 130$

480 вC

Charcoal from same site as RL-219. Scattered charcoal throughout Unit $\mathrm{D}_{2}$ at Loc VII in Meadow Valley Wash. Unit $\mathrm{D}_{2}$ is contained wholly within Unit D, stratigraphically 4th depositional unit in Meadow Valley Wash alluvium.

\section{RL-221. Etna Midden No. 1}

$20,000 \pm 400$

Pine needles (Pinus aristata, Pinus flexilis) from cliff alcove ca $100 \mathrm{~m}$ up E canyon wall; ca $9 \mathrm{~km} \mathrm{~S}$ of Caliente $\left(37^{\circ} 33^{\prime} 30^{\prime \prime} \mathrm{N}, 114^{\circ} 34^{\prime} 10^{\prime \prime} \mathrm{W}\right)$. 
Needles are from fossil woodrat midden in a rock crevice overlooking Meadow Valley Wash.

\section{RL-222. Stine Midden No. 1}

$14,980 \pm 250$

Dung (Neotoma sp) from cliff alcove ca $150 \mathrm{~m}$ up NW canyon wall in Stine canyon. Stine canyon is Meadow Valley Wash tributary which meets wash ca $17.4 \mathrm{~km} \mathrm{~S}$ of Caliente. Site is ca $1.6 \mathrm{~km}$ upstream from confluence $\left(37^{\circ} 27^{\prime} 50^{\prime \prime} \mathrm{N}, 114^{\circ} 36^{\prime} 40^{\prime \prime} \mathrm{W}\right)$. Dung is from fossil woodrat midden in rock crevice overlooking Stine canyon (Madsen, 1973).

\section{RL-223. Stine Midden No. 3}

$1770 \pm 90$

Dung (Neotoma sp) from fossil woodrat midden under rock overhang ca $125 \mathrm{~m}$ up NW canyon wall in Stine canyon. Coll site is same as for RL-222.

\section{0 вс}

Wood, probably willow (Salix sp) from Etna Cave, Lincoln Co, Nevada (37 $\left.35^{\prime} \mathrm{N}, 114^{\circ} 37^{\prime} \mathrm{W}\right)$. Sample above "3rd Floor" level (Wheeler, 1942). Coll 1935 by S M Wheeler; subm by D D Fowler. Comment (DDF): designated BP-3987 (Wheeler, 1942); accession No. 3336, Natl Park Service Visitors Center, Boulder City, Nevada, figurine was reportedly found stratigraphically below "Pleistocene age" horse dung in same level as Gypsum series projectile points. ${ }^{14} \mathrm{C}$ date agrees with dates on similar figurines from $\mathrm{N}$ Arizona.

\section{Joe's Valley Alcove series, Utah}

Wood charcoal from Joe's Valley Alcove, Emery Co, Utah $\left(39^{\circ} 17^{\prime} \mathrm{N}\right.$, $\left.111^{\circ} 16^{\prime} 30^{\prime \prime} \mathrm{W}\right)$. Coll 1972 and subm by E I DuBloois, U S Forest Service, Ogden, Utah. Comment (EID): discussion of samples in unpub preliminary repts, USDA Forest Service, Intermountain Region, Ogden, Utah.

RL-352. No. 1

$$
6760 \pm 180
$$

Single piece of wood charcoal from datum, $25 \mathrm{~cm} \mathrm{~N}$ of $4 \mathrm{~S}$ line $(375 \mathrm{~cm} \mathrm{~S}$ of datum), $201 \mathrm{~cm} \mathrm{E}$ of base line.

\section{RL-353. No. 2}

$6200 \pm 190$

Scattered charcoal coll over several sq $\mathrm{cm}$ of Bed I, Level 3, at $265 \mathrm{~cm}$ above datum and 300 to $350 \mathrm{~cm} \mathrm{~S}$ of datum.

RL-354. No. 13

Charcoal from contact of Bed I, Level 1 and shelter floor, $180 \mathrm{~cm}$ above datum, $400 \mathrm{~cm} \mathrm{~S}$ of datum. 
RL-355. No. 16

Charcoal from Bed I, Level 1 at $165 \mathrm{~cm}$ above datum, $580 \mathrm{~cm} \mathrm{~S}$ of datum, and $196 \mathrm{~cm} \mathrm{E}$ of base line.

RL-356. No. 17

$8210 \pm 220$

$6260 \mathrm{BC}$

Charcoal from Bed I, Level O, at $160 \mathrm{~cm}$ above datum, $535 \mathrm{~cm} \mathrm{~S}$ of datum, and $176 \mathrm{~cm} \mathrm{E}$ of base line.

\section{RL-413. Hogup Cave, Utah}

$1260 \pm 160$ AD 690

Phragmites arrow shaft fragment from $\mathrm{S}$ end of Hogup Mts, W of Great Salt Lake. Area is unmapped; closest approx is SW1/4, T8N, R12W. Sample, (\#24, Fs \#554-55), from Stratum 9, Hogup Cave (Aikens, 1970). Coll 1968 by C M Aikens; subm by D B Madsen, Div State History, Salt Lake City, Utah.

\section{Snowbird Bog series, Utah}

Unid. wood samples from possible forest floor mat at Snowbird, Little Cottonwood Canyon, Utah (40 30 $\left.0^{\prime} \mathrm{N}, 111^{\circ} 39^{\prime} 15^{\prime \prime} \mathrm{W}\right)$. Coll 1974 and subm by D B Madsen.

\section{RL-414. Snowbird Bog \#2}

$5600 \pm 190$

Sample from Unit IV-D.

3650 BC

\section{RL-415. Snowbird Bog \#3}

Sample from Unit II.

\section{Grand Canyon 671 series, Arizona}

Charcoal from structural timber, Grand Canyon Nat Monument, Mohave Co, Arizona (36 $\left.14^{\prime} \mathrm{N}, 113^{\circ} 09^{\prime} \mathrm{W}\right)$. Coll 1969, 1970 and subm by Richard Thompson, Southern Utah State Coll, Cedar City, Utah.

\section{RL-50. GC 671 No. 2}

Sample $.3 \mathrm{~m}$ deep under collapsed wall of basalt, Structure 1 .

RL-78. GC 671 No. 10

Sample $.3 \mathrm{~m}$ deep under collapsed wall of basalt, Structure 3 .

\section{RL-79. GC 671 No. 19}

$780 \pm 105$

Sample .5m deep under collapsed wall of basalt, Structure 3 .

\section{RL-80. GC 671 No. 21}

$840 \pm 110$

Sample .5m deep under collapsed wall of basalt, Structure 3. General Comment (RT): RL-80 age agrees with accepted occupation of area by 
Virgin Branch of Kayenta Anasazi; RL-50 and -79 ages suggest possibility of later occupation, while RL-78 age is considerably later than accepted date for end of occupation of area by Virgin Branch of Kayenta Anasazi.

\section{RL-224. Pinal County, Arizona}

Post from prehistoric ramada-like structure, likely Ironwood (Olneya tesota) but could possibly be Mesquite (Prosopis juliflora), from $S 2.4 \mathrm{~km}$ of Pinal Co, Sec 36, T10S, R4E, USGS Silver Reef Mts 15' quad, Arizona. Sample, No. 9, from SE posthole of Feature 1, AZ AA:5:4 (ASU) (Goodyear \& Dittert, 1973). Coll 1972 by A C Goodyear and Mark Raab; subm by A C Goodyear, Arizona State Univ, Tempe. Comment (ACG): date seems consistent with a late plain ware assoc with Sells phase and with structure dated by this sample. It is architecturally similar to other nearby Sells phase ramada-type houses.

\section{RL-397. Westwing site, Arizona}

$1090 \pm 90$

Charcoal from $W$ bank of Agua Fria R in central Arizona, Maricopa Co $\left(33^{\circ} 42^{\prime} 22^{\prime \prime} \mathrm{N}, 112^{\circ} 18^{\prime} 18^{\prime \prime} \mathrm{W}\right)$, within shallow pit oven 40 to $50 \mathrm{~cm}$ below present land surface (Weaver, 1974). Coll 1973 and subm by D E Weaver, Jr, Arizona State Univ, Tempe. Comment (DEW): sample probably represents beginning of occupation of Westwing site, a small Sedentary period Hohokam settlement.

\section{Central United States}

\section{Helb series, South Dakota}

Charcoal from Helb site, Campbell Co, South Dakota $\left(45^{\circ} 47^{\prime} 09^{\prime \prime} \mathrm{N}\right.$, $\left.100^{\circ} 20^{\prime} 55^{\prime \prime} \mathrm{W}\right)$. Subm by C R Falk, Midwest Archeol Center, Lincoln, Nebraska (Falk \& Calabrese, 1973).

\section{RL-298. 39CA208-104}

$940 \pm 90$

Wood charcoal from Feature 1, Level 7; extramural trash-filled pit adjacent to House 3 (705/710NW600). Coll 1972 by C R Falk. Comment (CRF): date consistent with NWU-38, -39, and -40 (unpub) and suggests early Extended Middle Missouri Variant in $\mathrm{S}$ portion of Cannonball Region of Middle Missouri Subarea.

\section{RL-299. 39CA208-360}

$430 \pm 90$

Wood charcoal (Prunus sp) from Feature 12, House 8; central hearth (220NW585). Coll 1973 by R K Nickel. Comment (CRF): date suggests use/occupation during late 15th or early 16th centuries AD and contrasts with dates of samples cited in comment, RL-298.

\section{Lower Grand (Davis) series, South Dakota}

Wood and charcoal from Lower Grand (Davis) site, Corson Co, South Dakota $\left(45^{\circ} 34^{\prime} 30^{\prime \prime} \mathrm{N}, 100^{\circ} 29^{\prime} \mathrm{W}\right)$. Subm by C R Falk. 
RL-300. 39CO14-1068

Wood charcoal (Salix/Populus spp) from Feature 102; extramural trash-filled pit adjacent to House 12 (640NW290). Coll 1969 by C R Falk.

\section{RL-301. 39CO14-1706}

$650 \pm 120$

Wood from Feature 402; wall post, House 12 (640NW350). Coll 1969 by S A Ahler.

\section{RL-302. 39CO14-1983}

Wood charcoal (Salix/Populus spp) from Feature 582, Level 3; extramural trash-filled pit adjacent to House 6 (450NW130). Coll 1969 by C R Falk.

\section{RL-303. 39CO14-2196}

Wood charcoal (Salix/Populus spp) from Test 6, adjacent to $\mathrm{N}$ fortification, Level 3. Coll 1969 by Steven Gentzler.

General Comment (CRF): samples suggest occupation of site during midto late-14th century AD, 2 centuries earlier than Lehmer's assignment of site to Extended Coalescent Variant of Middle Missouri tradition.

\section{Walth Bay series, South Dakota}

Charcoal and bone from Walth Bay site, Walworth Co, South Dakota $\left(45^{\circ} 24^{\prime} 45^{\prime \prime} \mathrm{N}, 100^{\circ} 16^{\prime} 30^{\prime \prime} \mathrm{W}\right)$. Subm by C R Falk.

\section{RL-304. 39WW 203-859}

$$
\begin{array}{r}
450 \\
\text { AD } 1500
\end{array}
$$

Wood charcoal (Salix/Populus spp) from Feature 116, burned structural (roof) debris; House 19 (670NW880), cultural Zone D. Coll 1971 by $S$ A Ahler.

\section{RL-305. 39WW 203-1437/1477}

$$
450 \pm 90
$$$$
\text { AD } 1500
$$

Wood charcoal (Salix/Populus spp) from Feature 257, combined Levels 5 and 6; intramural trash-filled pit assoc with House 9, cultural Zone D. Coll 1971 by S A Ahler.

\section{RL-306. 39WW 203-2207}

Wood charcoal (Salix/Populus spp) from Test 15, Feature 593, Level 7; extramural trash-filled pit adjacent to House 15, cultural Zone D. Coll 1971 by K A Lippencott.

\section{RL-307. 39WW203-2329/2340}

$$
\begin{array}{r}
310 \\
\text { AD } 1640
\end{array}
$$

Wood charcoal (Salix/Populus spp) from Test 16, Feature 741, combined Levels 4 and 6; extramural trash-filled pit adjacent to House 12, cultural Zone D. Coll 1971 by L G Madison. 
General Comment (SAA): 1st 4 samples in this series suggest a true age of mid- to late-15th century AD for Extended Coalescent Variant, cultural Zone D, occupation of site.

\section{RL-308. 39WW 203-2577}

$8030 \pm 1100$

Bone (Bison sp) from Sq 600NW990, 1.7m, cultural Zone B (Ahler et al, 1974). Coll 1972 by Danny Clinger. Comment (SAA): sample assoc with buried soil horizon, $\mathrm{IIA}_{1 \mathrm{~B}} / \mathrm{IIA}_{3 \mathrm{~B}}$, containing Middle period cultural remains. Sample very small; age based on bone collagen.

\section{RL-309. 39WW 203-970}

$7010 \pm 210$

Bone (Bison sp) adjacent to Test $10,1.5$ to $1.6 \mathrm{~m}$, cultural Zone A (Ahler et al, 1974). Coll 1971 by S A Ahler. Comment (SAA): sample assoc with buried soil horizon, IIIA $\mathrm{A}_{3 \mathrm{~B}}$, containing Late Paleo-Indian or early Archaic cultural materials. Age based on bone collagen.

\section{Eastern United States}

\section{Goddard site series, Maine}

Charcoal from area of Brooklin, Maine $\left(44^{\circ} 13^{\prime} 30^{\prime \prime} \mathrm{N}, 68^{\circ} 31^{\prime} 30^{\prime \prime}\right.$ W). Subm by B J Bourque, Maine State Mus, Augusta.

RL-368. No. 1

$3700 \pm 130$

$1750 \mathrm{BC}$

Charcoal in pit, assoc with ground slate point. Coll 1971 by Guy Mellgren.

RL-369. No. 3

$2300 \pm 120$

Charcoal in pit, assoc with 2 chipped and ground stone celts. Coll 1973 by Guy Mellgren.

\section{RL-177. Hudson River, New York}

$6150 \pm 120$

$4200 \mathrm{BC}$

Shell (Crasestrea virginica valve) from $\mathrm{E}$ bank, Hudson $\mathrm{R}$ opposite Pollepel I., $5 \mathrm{~km} \mathrm{~S}$ of Beacon, New York (41 $\left.27^{\prime} 18^{\prime \prime} \mathrm{N}, 73^{\circ} 58^{\prime} 42^{\prime \prime} \mathrm{W}\right)$. Sample from Stratum 3 at 38 to $51 \mathrm{~cm}$, underlying clayey sand, Stratum 2 , and overlying a hearth at $114 \mathrm{~cm}$, base of Stratum 3 (Ritchie, 1958). Coll ca 1951 by W A Ritchie; subm by W S Newman, Queens Coll, CUNY. Comment (WSN): charcoal from hearth below shells was dated, $4480 \pm 300$ (M-287; Science, 1958, v 127, p 1100). The nearly 2000-yr discrepancy between dates suggests hearth is either intrusive, or one of dates is wrong. Yet this is, by several hundred yrs, the oldest Hudson Oyster Shell Midden date known.

\section{RL-310. Hughes Farm site, West Virginia}

$$
1240 \pm 90
$$

Charcoal, No. 971, from Hughes Farm site, 46-Oh-9, Feature 13; Ohio Co, West Virginia $\left(40^{\circ} 08^{\prime} 46^{\prime \prime} \mathrm{N}, 80^{\circ} 38^{\prime} 00^{\prime \prime} \mathrm{W}\right)$ (Dunnell, 1962). Coll 1959 and subm by R C Dunnell, Univ Washington, Seattle. Com- 
ment (RCD): Feature 13, from which sample was taken, is part of late Watson/early Monogahela hamlet representing deepest part of occupation at 46-Oh-9. Assoc ceramics include ca 12\% of shell-tempered, previously assumed much later when compared chronologically with other areas.

\section{Deep Shelter series, Kentucky}

Carbonized wood from Deep Shelter rock shelter, S Rowan Co, Kentucky, at edge of and slightly into E Mts $\left(38^{\circ} 03^{\prime} \mathrm{N}, 83^{\circ} 27^{\prime} 30^{\prime \prime} \mathrm{W}\right)$ (Dorwin \& Warholic, 1970). Coll 1969 by J T Dorwin and D T Warholic; subm by J T Dorwin, Univ Kentucky, Lexington.

\section{RL-67. KY Ro 34, 1119}

$7240 \pm 550$

Small pieces of carbonized wood, scattered throughout fill of small pit, Feature 4, which was at edge of drip line of shelter at depth $1.32 \mathrm{~m}$ below datum to $1.63 \mathrm{~m}$. Pit is above and slightly SE of area from which RL-68 was obtained. Comment (JTD): date is for a general horizon of pit. Horizon included LeCroy Bifurcated Base points. RL-67 and -68 agree and fit well within chronology and projectile point sequence of St Albans, West Virginia site (Broyles, pers commun).

\section{RL-68. KY Ro 34, 1120}

$8520 \pm 470$

Small pieces of carbonized wood from upper level of a remnant living floor of shelter, which contained 4 separate superimposed layers consisting of ashy sandy soil, bone and chert debitage. Sufficient charcoal for dating from lower levels was unavailable. Sample is from ca $40 \mathrm{~cm}$ below top of pit containing RL-67, and slightly NW. Comment (JTD): date is in keeping with stratigraphy at Deep Shelter and artifact sequence established at St Albans site, West Virginia. General horizon of date contained Charleston Corner Notched points, dated slightly earlier by Broyles (pers commun).

\section{Mayo series, Kentucky}

Samples from Mayo site, 15-Jo-14, on $\mathrm{T}^{2}$ of Paint Creek, ca $1.6 \mathrm{~km}$ from its confluence with Levisa Fork on Big Sandy R W of Paintsville,

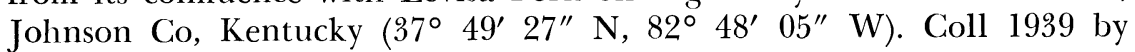
J C Greenacre; subm by R C Dunnell.

RL-311. 15-Jo-14/51

AD 1150

$800 \pm 100$

Charcoal from Level II, sheet midden O-O. Comment (RCD): should date early middle Woodside phase occupation of Levisa Fork Valley and is comparable to dates for early Fort Ancient in Ohio.

RL-322. 15-Jo-14"B"

$1060 \pm 90$

Animal scrap bone, (mostly Odocoileus viriginianus) from Feature 13, a sheet midden, probably surface deposit aboriginally, which is an 
integral part of Woodside component of site. Comment (RCD): should be relatively close to RL-311, as they both are part of same Woodside occupation; they reasonably agree, in that 15-Jo-14 is relatively early Woodside phase, suggested by stylistic analysis.

\section{RL-77. Peace Camp site, Florida}

Shell (Strombus gigas) from Peace Camp site, Broward Co, Florida $\left(26^{\circ} \mathrm{N}, 80^{\circ} 10^{\prime} \mathrm{W}\right)$. In stratigraphic excavation; Sq N-18, Stratum 6, $1.3 \mathrm{~m}$ below datum, and just below deepest Norwood Plain and St Johns Incised pottery. Coll 1969 by Wilma Williams; subm by R P Bullen, Florida State Mus, Gainesville. Comment (RPB): dates 1st occupancy of site (Wilma Williams, ms in preparation, Broward Co Arch Soc).

\section{Lanoraie series, Quebec}

\section{E. Canada}

Charcoal from Lanoraie, Berthier Co, Quebec $\left(45^{\circ} 58^{\prime} \mathrm{N}, 73^{\circ} 13^{\prime} \mathrm{W}\right)$. Coll 1970 and subm by Georges Barré, Min Affaires culturelles, Quebec.

\section{RL-178. La-6}

$$
580 \pm 90
$$

From base of pit containing charcoal, potsherds, and carbonized corn kernels.

\section{RL-179. La-9}

$550 \pm 105$

From base of pit containing charcoal and potsherds.

General Comment (HT): based on body treatment and decorative techniques, Lanoraie site initially was assigned to early stage of Iroquois Prehistory (MacNeish, 1952; Wright, pers commun). These 2 dates indicate that chronologic significance of such attributes should be re-evaluated in central St Lawrence Valley area.

\section{Cap-Chat series, Quebec}

Charcoal from Cap-Chat site, Gaspé Nord Co, Quebec $\left(49^{\circ} 10^{\prime} \mathrm{N}\right.$, $\left.66^{\circ} 40^{\prime} \mathrm{W}\right)$. Subm by Georges Barré.

\section{RL-180. DgDq-1}

$1330 \pm 105$

Sample from S sec of lowest cultural layer of site. Coll 1971 by Georges Barré.

\section{RL-181. DgDq I:43.1}

$1410 \pm 105$

Sample from N sec of lowest cultural layer of site. Coll 1971 by Hughette Trudeau.

\section{RL-343. Restigouche River, New Brunswick}

$$
2030 \pm 130
$$

Charcoal, C1Dq-1:C11, from S shore of Restigouche R, Old Church Point, near Atholville, Restigouche Co, New Brunswick (47 $59^{\prime} 35^{\prime \prime} \mathrm{N}$, $66^{\circ} 44^{\prime} 20^{\prime \prime} \mathrm{W}$ ), from pit dug into sterile gravels, assoc with pottery 
fragments, stemmed projectile point, and fire-cracked rock. Coll 1972 by K Crandall; subm by C J Turnbull, Hist Resources Admin, Prov New Brunswick. Comment (CJT): site may be multi-component occupation, most recently, 18th century. Sample may date earliest settlement.

\section{RL-344. Northumberland County, New Brunswick}

Charcoal, CfDw-2:1, from junction of Little Southwest Miramichi R and Northwest Miramichi R, near Red Bank, Northumberland Co, New Brunswick $\left(46^{\circ} 51^{\prime} 00^{\prime \prime} \mathrm{N}, 67^{\circ} 50^{\prime} 00^{\prime \prime} \mathrm{W}\right)$, assoc with burials in probable burial mound $12 \mathrm{~m}$ across, $1.2 \mathrm{~m}$ high. Subm by C J Turnbull. Comment (CJT): site is a problem: burial mound, and some artifacts indicate early Woodland assoc; exceptional preservation of wood, bark cloth, and bones indicate much more recent date. Because site was dug by an amateur, assocs are still tentative. Only $1 / 2$ sample was submitted.

\section{RL-345. Old MacDonald Farm, New Brunswick}

Charcoal, CgDi-1:334, from Old MacDonald Farm, W side of Bartibog $\mathrm{R}, .6 \mathrm{~km}$ downriver from bridge $\left(47^{\circ} 05^{\prime} 40^{\prime \prime} \mathrm{N}, 65^{\circ} 21^{\prime} 15^{\prime \prime} \mathrm{W}\right)$. Sample from hearth, beneath plough zone. Coll 1972 and subm by $\mathrm{C}$ J Turnbull. Comment (CJT): only sample from an aceramic component.

\section{F. West Indies}

\section{Grenadines series, West Indies}

Samples coll 1970 and subm by R P Bullen, Florida State Mus, Gainesville (Bullen \& Bullen, 1972).

\section{RL-70. FSM-BF No. 8}

$1470 \pm 110$

Shell (Strombus gigas) from Chatham Bay, Union I., Grenadines $\left(12^{\circ} 35^{\prime} 56^{\prime \prime} \mathrm{N}, 61^{\circ} 27^{\prime} 08^{\prime \prime} \mathrm{W}\right)$, from narrow zone ca $20 \mathrm{~cm}$ below surface; a 1-period deposit. Comment (RPB): dates a Modified Saladoid ceramic complex including Troumassee Decorated Cylinders.

\section{RL-71. FSM-BF No. 9} AD 1420

$530 \pm 110$

Shell (Strombus gigas) from Banana Bay, Belliceaux I. $\left(12^{\circ} 57^{\prime} \mathrm{N}\right.$, $\left.61^{\circ} 09^{\prime} 05^{\prime \prime} \mathrm{W}\right)$, from top of midden in eroded face of site. Comment (RPB): this is a check on RL-27 (R, 1971, v 13, p 76), from middle part of midden, dated, AD $1230 \pm 100$. Range is reasonable. Clearly, site is prehistoric, at least for midden.

\section{St Vincent series, W est Indies}

Samples coll by Earle Kirby and (RL-73) R P Bullen; subm by R P Bullen (Bullen \& Bullen, 1972).

RL-72. FSM-BF No. 10 $370 \pm 110$

West Indian Top shells (Pica) from Indian Bay, SE St Vincent $\left(13^{\circ}\right.$ $08^{\prime} 10^{\prime \prime} \mathrm{N}, 61^{\circ} 12^{\prime} 45^{\prime \prime} \mathrm{W}$ ), from below pottery-producing midden. Com- 
ment (RPB): shells below midden did not represent preceramic occupation. Midden bleongs to proto-historic Suazey complex and shells date midden occupation.

\section{RL-73. FSM-BF No. 11}

$1670 \pm 160$

Charcoal from Lower Buccament Valley in front of cave $\left(13^{\circ} 10^{\prime} \mathrm{N}\right.$, $\left.61^{\circ} 16^{\prime} \mathrm{W}\right)$, from test at depth $1.2 \mathrm{~m}$. Comment (RPB): dates early Modified Saladoid ceramics of area.

\section{RL-74. FSM-BF No. 12}

$930 \pm 110$

Charcoal from Fitz Hughes site, NW St Vincent $\left(15^{\circ} 17^{\prime} 10^{\prime \prime} \mathrm{N}, 61^{\circ}\right.$ $14^{\prime} \mathrm{W}$ ), from deep pit leading down from occupation zone. Overburden and top of midden removed by bulldozer. Comment (RPB): dates a mixed Suazey-Caliviny ceramic complex.

\section{RL-75. FSM-BF No. 13}

$1540 \pm 110$ AD 410

Charcoal from Arnos Vale (swamp) near airport, SE St Vincent $\left(13^{\circ}\right.$ $08^{\prime} 20^{\prime \prime} \mathrm{N}, 61^{\circ} 13^{\prime} 00^{\prime \prime} \mathrm{W}$ ), from lower artifact-producing zone under ca $2.4 \mathrm{~m}$ water and volcanic deposited material. Comment (RPB): dates spectacular Modified Saladoid pottery from this low zone. Pottery seems younger than that from Kingstown Post Office site, RL-28 (R, 1971, v 13, p 76), dated, AD $160 \pm 100$. Indicates typologic changes during Modified Saladoid period.

\section{RL-76. Savanne Suazey site, Grenada}

$550 \pm 110$

Shell (Strombus gigas), FSM-BF No. 14, from Savanne Suazey site, NE Grenada $\left(12^{\circ} 10^{\prime} \mathrm{N}, 61^{\circ} 37^{\prime} \mathrm{W}\right)$, from burial area with Suazey series pottery, iron fragments, and 2 Spanish Olive jar sherds. Burials were in shallow midden deposit, 0 to $38 \mathrm{~cm}$ from surface to base of deposit (Bullen, 1964). Coll 1970 and subm by R P Bullen. Comment (RPB): indicates burials are proto-historic and Suazey ceramic complex lasted until, if not into, Post-Columbian times.

\section{RL-155. Couronue site, West Indies} AD 1170

$$
780 \pm 100
$$

Shell (Strombus gigas) from E coast of Guadeloupe $\left(12^{\circ} 30^{\prime} \mathrm{N}, 61^{\circ}\right.$ $30^{\prime} \mathrm{W}$ ), just below surface in a 1-period site. Coll 1971 and subm by $\mathrm{R} P$ Bullen. Comment (RPB): site produced Suazey ceramics, correlated with Carib occupancy. Date is consistent, possibly a bit early.

\section{RL-156. Vivé, Martinique}

$1730 \pm 110$

Charcoal from NE coast of Martinique, French West Indies $\left(14^{\circ} 51^{\prime}\right.$ $\mathrm{N}, 61^{\circ} 05^{\prime} \mathrm{W}$ ), from lower cultural zone below sterile volcanic tuff. Coll 1971 and subm by R P Bullen. Comment (RPB): dates a very late phase of Insular Saladoid ceramic tradition (or perhaps very early phase of Modified Saladoid tradition). Date is consistent with others: S-85 (unpub) 
from volcanic tuff was dated, AD $295 \pm 150$; UGA-113 (unpub) from upper cultural zone overlying volcanic tuff was dated, AD $420 \pm 75$.

\section{GEOLOGIC SAMPLES}

\section{A. North America}

RL-171. Utah Lake, Utah

$11,400 \pm 850$

Peat from Utah Lake, Utah Co $\left(40^{\circ} 15^{\prime} \mathrm{N}, 111^{\circ} 45^{\prime} \mathrm{W}\right)$, from ca $1.6 \mathrm{~m} \mathrm{~W}$ of shoreline adjacent to Geneva steel mills, at depth 490 to $500 \mathrm{~cm}$. Coll 1970 and subm by R F Bolland, Univ of Utah, Salt Lake City. Comment (RFB): cores were taken for study on diatoms of Utah Lake.

\section{RL-157. Orbach Mall, New York}

$13,470 \pm 850$

Peat and silt from foundation excavation for Orbach Queens Fashion Mall on Queens Blvd near Long Island Expwy, Rego Park, Queens, New York $\left(40^{\circ} 44^{\prime} 00^{\prime \prime} \mathrm{N}, 73^{\circ} 52^{\prime} 24^{\prime \prime} \mathrm{W}\right)$, at depth ca $7 \mathrm{~m}$, near top of stratified and laminated silt of glacial Lake Flushing, overlain by .3 to $1.5 \mathrm{~m}$ till, probably Sirkin's "upper till", his Roslyn Till equivalent, the latest evidence of glacial advance to Long I. (Sirkin, 1971). Coll 1971 by M I Esrig; subm by W S Newman, Queens Coll, CUNY. Comment (WSN): 1st intertill date from Long I. and surprisingly young. Sirkin believed minimum age of Roslyn Till > 17,000 yr.

\section{RL-318. Montauk Point, New York \\ $\mathbf{3 8 , 0 0 0} \underset{-3200}{+5600}$}

Wood from $1.6 \mathrm{~km} \mathrm{SW}$ of Montauk Point in S shore bluff, Long I., New York $\left(41^{\circ} 03^{\prime} 12^{\prime \prime} \mathrm{N}, 71^{\circ} 52^{\prime} \mathrm{W}\right)$, from clayey silt lenses containing peat and wood, lying between 2 layers of till, upper ca $3 \mathrm{~m}$ thick and lower $1.2 \mathrm{~m}$ thick (Perlmutter \& De Luca, 1963). Coll 1972 by R E Matarese; subm by W S Newman. Comment (WSN): pollen analyses suggest tundra/Boreal forest/tundra sequence. Pollen data plus old age of sample indicate a Wisconsin Interstadial.

\section{RL-24.5. Gross Bog, Connecticut}

$$
12,750 \pm 230
$$

Gyttja peat from bog at $330 \mathrm{~m}$ alt, NW flank of Housatonic Highlands, NW Connecticut $\left(41^{\circ} 48^{\prime} \mathrm{N}, 73^{\circ} 29^{\prime} 26^{\prime \prime} \mathrm{W}\right)$, from $6 \mathrm{~m}$ beneath bog surface at gyttja-clay interface (Kelley, ms in preparation). Coll 1972 by G C Kelley; subm by W S Newman. Comment (WSN): dates T (for tundra) Zone of Gross Bog.

\section{B. South America}

\section{RL-113. Puerto Varas, Chile}

$$
16,270 \pm 360
$$

Wood from bank on $\mathrm{S}$ side of rd leading W from Puerto Varas to Panamerican Hwy, under rr bridge $\left(41^{\circ} 18^{\prime} \mathrm{S}, 72^{\circ} 59^{\prime} \mathrm{W}\right) ; 160 \mathrm{~cm}$ gyttja covered with peat lenses and fragments of wood, overlying gravel; sample $20 \mathrm{~cm}$ above base of gyttja. This organic sequence is covered by $14 \mathrm{~m}$ lake sediment; uppermost peat is thus in similar stratigraphic posi- 
tion to peat exposed in Calle Rosa, Puerto Varas, dated (uppermost peat) at 14,820 \pm 230 (I-5033, unpub) (Mercer, 1972). Coll 1971 and subm by J H Mercer, Inst Polar Studies, Ohio State Univ, Columbus. Comment (JHM): deposition of gyttja began at start of low-water phase of Lago Llanquihue, when glacial recession opened outlet to tidewater Seno Reloncavi at $\mathbf{E}$ end of lake. Sample age is, thus, minimum for start of Varas Interstadial. It is consistent with age of I-5033, and similar to date of early part of the Erie Interstadial in North America.

RL-116. Frutillar End Moraine, Chile

$20,100 \pm 550$

Peat from gravel pit on $\mathrm{S}$ side of $\mathrm{rd}$ from Frutillar Alto to Frutillar

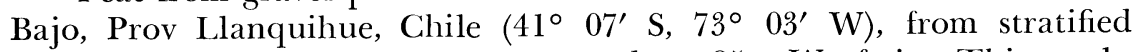
drift below thin compact till that extends ca $25 \mathrm{~m} \mathrm{~W}$ of site. This marks outer limit of maximum stand of Lago Llanquihue glacier (Mercer, 1972). Coll 1971 and subm by J H Mercer. Comment (JHM): peat was fibrous and unhumified, probably derived from near surface of bog overridden by ice, near its maximum stand. Date agrees well with I-5679: $19,450 \pm 400$ (unpub) at surface of bog beneath end moraine at adjacent Lago Rupanco.

RL-120. Lago Llanquihue, Chile

$17,370 \pm 670$

Peat, $3 \mathrm{~km} \mathrm{~N}$ of Puerto Octay, Prov Osorno, Chile; $50 \mathrm{~m} \mathrm{~W}$ of Rte U-55-V crossing of Río Chan-Chan $\left(40^{\circ} 57^{\prime} \mathrm{S}, 72^{\circ} 50^{\prime} \mathrm{W}\right)$, from $3 \mathrm{~m}$ depth in old outlet of Lago Llanquihue, now ca $100 \mathrm{~m}$ above lake level. Obtained by Hiller borer from contact with material thought to be cobbles (Mercer, 1972). Coll 1971 by C J Heusser; subm by J H Mercer. Comment (JHM): outlet drained ice-marginal lake when Lago Llanquihue glacier reached lake shore moraines further S. Date agrees with RL-116, above, and I-5679 (unpub) which suggest a glacial maximum ca 19,400 BP. Outlet apparently cuts through moraines of earlier, greater advance of last major glaciation.

RL-119. Moreno Glacier, Argentina

$9510 \pm 210$

Peat (mainly Juncus) from depression from head of Bahía Catalán of Lago Rico, and Lago Argentino, Prov Santa Cruz, Argentina $\left(50^{\circ} 29^{\prime}\right.$ $\left.\mathrm{S}, 73^{\circ} 02^{\prime} \mathrm{W}\right)$. Long narrow bog, surface $42 \mathrm{~m}$ above Lago Argentino, occupies depression. Sample is basal peat from bog. Coll 1971 and subm by $\mathrm{J} \mathrm{H}$ Mercer. Comment (JHM): sample invalidates conclusions drawn from data of I-2201: $3830 \pm 115$ (Mercer, 1968), which was misidentified as basal peat for this same section of bog. Site is $800 \mathrm{~m}$ from present ice front; date is minimum for shrinkage of Moreno Glacier close to its present dimensions; this agrees with age of basal peat in ancient outlet of Lago Rico: 10,000 \pm 140, I-2209 (Mercer, 1968).

$$
\text { C. Europe }
$$

RL-164. Helice site, Greece

$1310 \pm 110$

Assorted shells, urchins near Aigion, Greece, $24 \mathrm{~km} \mathrm{E}$, at supposed site of ancient Helice; W of mouth of Selinous R, Bay of Corinth in $44 \mathrm{~m}$ 
water, offshore from cement markers at Volimeka $\left(38^{\circ} 17^{\prime} 18^{\prime \prime} \mathrm{N}, 22^{\circ}\right.$ $08^{\prime} 42^{\prime \prime}$ E). Sample from sediments in lower $20 \mathrm{~cm}$ of core; $5 \mathrm{~cm}$ diam, $2 \mathrm{~m}$ long (Marinatos, 1960; 1970). Coll 1971 by S N Marinatos, H E Edgerton, and Peter Throckmorton; subm by $\mathrm{H}$ E Edgerton, Massachusetts Inst Technol, Cambridge. Comment (HEE): date implies material from $2 \mathrm{~m}$ below base was deposited after earthquake of $325 \mathrm{BC}$; other disturbances occurred since then. More radiocarbon dates from greater depths will establish geologic sequence of sediments.

\section{OCEANOGRAPHIC SAMPLES}

\section{Core V29-183K series, North Atlantic}

Deep sea sediment samples containing planktonic forams from a core under $3492 \mathrm{~m}$ water, North Atlantic Ocean $\left(49^{\circ} 08^{\prime} \mathrm{N}, 25^{\circ} 30^{\prime} \mathrm{W}\right)$. Coll 1972 and subm by Andrew McIntyre, Lamont-Doherty Geol Observatory, Palisades, New York.

RL-212. V29-183K

$3860 \pm 130$

From 7 to $8 \mathrm{~cm}$ depth.

RL-213. V29-183K

$5440 \pm 130$

From 14.5 to $15.5 \mathrm{~cm}$ depth.

RL-214. V29-183K

$8950 \pm 220$

From 20.5 to $21.5 \mathrm{~cm}$ depth.

RL-215. V29-183K

$15,950 \pm 620$

From 25 to $26 \mathrm{~cm}$ depth.

REFERENCES

Ahler, S A, Davies, D K, Falk, C R, and Madsen, D B, 1974, Holocene stratigraphy and archeology in the Middle Missouri River Trench, South Dakota: Science, V 184, p $905-908$.

Aikens, C M, 1970, Hogup Cave: Univ Utah Anthropol Papers, no. 93.

Bullen, R P, 1964, The archaeology of Grenada: Contr Florida State Mus, Social Sciences, no. 11, p 5-17.

Bullen, R P and Bullen, A K, 1972, Archaeological investigations on St Vincent and the Grenadines West Indies: Wm L Bryant Foundation, Am Studies report no. 8.

Crane, H R and Griffen, J B, 1958, University of Michigan radiocarbon dates II: Science, v 127, p 1098-1105.

Dorwin, J T and Warholic, D T, 1970, Archaeological investigation of the Deep Shelter, Rowan County, Kentucky: Rept to NPS.

Dunnell, R C, 1962, The Hughes Farm site (46 Oh 9), Ohio County, West Virginia: West Virginia Archaeol Soc, pub ser no. 7, Moundsville.

Falk, C R and Calabrese, F A, 1973, Helb: a preliminary statement: Plains Anthropologist, v 18, p 336-343.

Goodyear, A C and Dittert, A E, Jr, 1973, HECLA I: a preliminary rept on the archaeological investigations at the Lakeshore Proj Papago Reservation, South Central Arizona: Arizona State Univ, Dept Anthropol research paper 4.

Grabert, G F and Larsen, C E, 1973, Marine transgressions and cultural adaptation: preliminary tests of an environmental model: ICAES mtgs proc, Sept 1973.

Kelley, G C, PhD dissert, Syracuse Univ, and USGS quad repts, in preparation.

MacNeish, R S, 1952, Iroquois pottery types: National Mus Canada Bull no. 124, Ottawa.

Madsen, D B, 1973, Late Quaternary paleoecology in the Southeastern Great Basin: PhD dissert, Dept Anthropol, Univ Missouri. 
Marinatos, S N, 1960, Helice: a submerged town of classical Greece: Archacology, v 13, p 186-193.

1970, Investigation in Helice: Acta Acad Athens, v 41, p 511-518.

Mercer, J H, 1968, Variations of some Patagonian glaciers since the Late-Glacial: Am Jour Sci, v 266, p 91-109.

1972, Chilian glacial chronology 20,000 to 11,000 carbon-14 years ago: some global comparisons: Science, v 176, p 1118-1120.

Noakes, J E, Kim, S M, and Stipp, J J, 1966, Chemical and counting advances in liquid scintillation age dating: 6th internatl conf on radiocarbon and tritium dating,
Proc, USAEC Conf-650652, Pullman, Washington.

Perlmutter, N M and DeLuca, F A, 1963, Availability of fresh ground water, Montauk Point area, Suffolk County, Long Island, New York: USGS Water-Supply Paper 1613-B, p 39. Richie, W A, 1958, An introduction to Hudson Valley prehistory: New York State
Mus Bull 367.

Sirkin, L A, 1971, Surficial glacial deposits and postglacial pollen stratigraphy in central Long Island, New York: Pollen et Spores, v 13, p 94-100.

Tucek, C S, 1971, Radiocarbon, Ltd natural radiocarbon measurements I: Radiocarbon, v 13, p 74-77.

Weaver, D E, Jr, 1974, Archacological investigations at the Westwing site AZ T:7:07 (ASU), Agua Fria River Valley, Arizona: Arizona State Univ Dept Anthropol research papers, no. 7 , Tempe.

Wheeler, S M, 1942, The archaeology of Etna Cave, Lincoln County, Nevada: repr 1973: Desert Research Inst Pubs in Soc Sci, no. 7, Reno. 